\title{
ANALYTICAL AND NUMERICAL ANALYSIS OF THE GENERALIZED SHKAROFSKY FUNCTION
}

\author{
N. M. TEMME \\ Centrum voor Wiskunde en Informatica, Amsterdam. The Netherlands
}

A. E. SUMNER and S. S. SAZHIN

Department of Physics, The University of Sheffield, England, U.K.

(Received 1 August, 1991)

\begin{abstract}
The main analytical properties of the generalized Shkarofsky function and a numerical code for its computation are discussed. The results of a numerical analysis are compared with the results of an asymptotic analysis for parameter values relevant to the problem of whistler-mode propagation in the Earth's magnetosphere. This comparison allows us to specify the range of applicability of different approximations to the generalized Shkarofsky function, which have been used for the analysis of relativistic effects on whistler-mode propagation and instability.
\end{abstract}

\section{Introduction}

In our previous paper (Sazhin and Temme, 1990; hereafter referred to as ST), we presented an asymptotic analysis of relativistic effects on parallel whistler-mode propagation and instabilities based on the equation

$$
N^{2}=1-\frac{2 X}{r}\left[\mathscr{F}_{1 / 2,2}-\frac{\mathrm{d} \cdot \bar{F}_{3 / 2,2}}{\mathrm{~d} z}\left(A_{e}-1\right) N^{2}\right],
$$

where

$$
\mathscr{F}_{q, p} \equiv \mathscr{F}_{q, p}(z, a, b)=-i \int_{0}^{x} \exp \left[i z t-\frac{a t^{2}}{1-i t}\right](1-i t)^{-q}(1-i b t)^{-p} \mathrm{~d} t,
$$

$z=2(1-Y) / r ; \quad a=N^{2} / r ; \quad r=p_{0 \|}^{2} /\left(m_{e}^{2} c^{2}\right) ; \quad b=A_{e} ; \quad X=\Pi_{0}^{2} / \omega^{2} ; \quad Y=\Omega_{0} / \omega ;$ $A_{e}=p_{0 \perp}^{2} \mid p_{0 \|}^{2} ; \Pi_{0}, \Omega_{0}$, and $\omega$ are the electron plasma frequency at rest, the electron gyrofrequency at rest and the wave frequency, respectively, $N$ is the wave refractive index, $m_{e}$ is the electron mass at rest, $c$ is the velocity of light.

When deriving (1) we assumed an electron distribution function of the form

$$
f\left(p_{\perp}, p_{\|}\right)=\left(j ! \pi^{3 / 2} p_{0 \perp}^{2 j+2} p_{0 \|}\right)^{-1} p_{\perp}^{2 j} \exp \left(-\frac{p_{\perp}^{2}}{p_{0 \perp}^{2}}-\frac{p_{\|}^{2}}{p_{0 \|}^{2}}\right),
$$

where $p_{0 \perp(\|)}$ is the electron thermal momentum in the direction perpendicular (parallel) to the magnetic field, $p_{\perp}$ and $p_{\|}$are the electron momenta in the corresponding directions, $j=0,1,2, \ldots$ 
We restricted our analysis to the case $j=0$ (bi-Maxwellian plasma). The generalization to $j \neq 0$ would be straightforward (Tsai et al., 1981; Sazhin, 1989). We also restricted ourselves to considering wave frequencies well above the proton gyrofrequency, so that the protons and other heavy ions could be considered as a neutralizing background. Finally we assumed the plasma to be weakly relativistic so that $p_{0 \perp(\|)} \ll m_{e} c$.

We call the function $\mathscr{F}_{4, p}$ the generalized Shkarofsky function. It is related to the conventional Shkarofsky function (Shkarofsky, 1966; Robinson, 1986, 1987)

$$
\mathscr{F}_{q}(z, a)=-i \int_{0}^{x} \exp \left[i z t-\frac{a t^{2}}{1-i t}\right](1-i t)^{-q} \mathrm{~d} t
$$

by the identities

$$
\begin{aligned}
& \mathscr{F}_{q, 0}(z, a, b)=\widetilde{\mathscr{F}}_{q, p}(z, a, 0)=\mathscr{F}_{q}(z, a), \\
& \widetilde{\mathscr{F}}_{q, p}(z, a, 1)=\widetilde{\mathscr{F}}_{q+p}(z, a) .
\end{aligned}
$$

In view of (1) the analysis of relativistic effects on parallel whistler-mode propagation and instabilities reduces to the corresponding analysis of the generalized Shkarofsky function $\widetilde{F}_{q, p}$. ST based their consideration of these effects on an asymptotic analysis of $\overline{\mathscr{F}}_{q, p}$ valid for values of $a$ satisfying $a \gg 1$. This holds true in most cases of whistlermode propagation in the Earth's magnetosphere. However, the results of this asymptotic analysis, as well as those of other similar analyses, cannot be rigorously justified unless we compare them directly with numerical results. Such a comparison is made in this paper.

In Section 2 we summarize the main analytical properties of the generalized Shkarofsky function. A summary of the results of the asymptotic analysis of $\bar{F}_{q, p}$ is given in Section 3. In Section 4 we describe the numerical code on which we base our numerical analysis of $\mathscr{F}_{q, p}$. In Section 5 we compare numerical and analytical results for $p=2$ and $q=0.5$ and $q=1.5$ (see Equation (1)) and for values of $a$ and $b$ realistic for the Earth's magnetosphere. The main results of the paper are summarized in Section 6.

\section{Analytical Properties of $\mathscr{F}_{q, p}$}

Following ST and after some minor rearrangements we rewrite $\mathscr{F}_{q, p}$ defined by (1) in the form

$$
\mathscr{F}_{q, p}=\int_{0}^{-i x} e^{-(z-a) s-a s /(1+s)} f(s) \mathrm{d} s,
$$

where

$$
f(s)=(1+s)^{-q}(1+b s)^{-p} .
$$


Integral (7) converges at $s=-i \propto$ if

$$
\mathfrak{F}(z-a)>0 .
$$

For other values of $z-a$ we use analytic continuation.

\subsection{Analytic Continuation of $\widetilde{\mathscr{F}}_{q, p}$}

For the analysis of $\pi_{q, p}$ it is convenient to introduce the variable

$$
\zeta=z-a \text {. }
$$

Following the condition (9) we initially assume that

$$
0<\arg \zeta<\pi .
$$

The function can be analytically continued to the half plane $\mathfrak{R} \zeta>0$ by directing the path of integration in (7) along the real positive axis: i.e.,

$$
\mathscr{F}_{q, p}=\mathscr{F}_{q, p}(\zeta, a)=\int_{0}^{x} e^{-\zeta s-a s /(1+s)} f(s) \mathrm{d} s,
$$

where $\Re \zeta>0$, that is $-\pi / 2<\arg \zeta<\pi / 2$. Note that we have changed our notation slightly: $\tilde{F}_{q, p}(\zeta, a) \equiv \tilde{F}_{q, p}(z, a, b)$, with $\zeta=z-a$.

Now the function is defined in terms of real positive $s$, and the function is real when $\zeta$ is real and positive and when $a$ is real $(p, q, b$ are assumed to be real). We have the following conjugation rules:

$$
\begin{aligned}
& \mathscr{F}_{q, p}(\bar{\zeta}, a)=\overline{\bar{F}_{q, p}(\zeta, a)}, \quad a \text { real }, \\
& \bar{F}_{q, p}(\zeta, \bar{a})=\overline{\mathscr{F}_{q, p}(\zeta, a)}, \quad \zeta>0 .
\end{aligned}
$$

Analytic continuation to the half plane $₹ \zeta<0$ follows from turning the path of integration in (11) along the positive imaginary axis in the upper half plane ( $\& s>0)$. Thus

- we obtain the function $\mathscr{F}_{q, p}(\zeta, a)$ as an analytic function in the principal sector

$$
-\pi<\arg \zeta \leq \pi, \quad|\zeta| \neq 0
$$

$a$ being an arbitrary complex number. (By turning the path of integration quite close to the negative real $s$-axis, we may extend the principal sector to $-3 \pi / 2<\arg \zeta<3 \pi / 2$; however, remembering physical applications to whistler-mode waves, in what follows we will be interested mainly in the sector $-\pi<\arg \zeta \leq \pi$.)

In view of the further applications to the problem of whistler-mode propagation in the magnetosphere, modulae of $p, q$, and $b$ are assumed to be less than, say, 5 .

$z$ and $a$ are complex (and, hence, $\zeta$ may be complex), with $\Re z<0, \Re a>0$. The imaginary parts of both $a$ and $z$ are not large, say $\max (\mathfrak{F} z, \mathfrak{F} a)<10$, but there are no bounds on the values of $\Re a$ and $\Re z$. 


\subsection{RECURSION RELATIONS FOR $\tilde{F}_{q, p}$}

The following recursion relations for $\bar{F}_{q, p}$ defined by (11) are valid:

$$
\begin{aligned}
& (b-1) \cdot \bar{F}_{q, p}=b \cdot \bar{F}_{q-1, p}-\bar{F}_{q, p-1}, \\
& \zeta \tilde{F}_{q, p}=1-a \cdot \widetilde{F}_{q+2, p}-q \widetilde{\mathscr{F}}_{q+1, p}-p b \widetilde{F}_{q, p+1}, \\
& b \zeta \overline{\mathscr{F}}_{4-1, p}=1+a(b-1) \cdot \widetilde{F}_{q+2, p}+[q(b-1)-a b] \cdot{\widetilde{F_{q}}}_{q+1, p}+ \\
& +[\zeta(b-1)-q h-(p-1) b] \mathscr{F}_{q, p} .
\end{aligned}
$$

Furthermore, we have

$$
\frac{\mathrm{d} \mathscr{F}_{q, p}}{\mathrm{~d} \zeta}=\frac{\mathrm{d} \mathscr{F}_{q, p}}{\mathrm{~d} z}=\mathscr{F}_{q, p}-\mathscr{F}_{q-1, p} .
$$

The recursion formulae (especially the inhomogeneous relations (14) and (15)) are useful in checking asymptotic or numerical approximations. The relation for the derivative in (16) follows from the integral (11). The same holds for (13). Relation (14) follows from (11) written in the form

$$
\zeta \mathscr{F}_{q, p}=-\int_{0}^{x} e^{-a s /(1+s)} f(s) \mathrm{d} e^{-\zeta s},
$$

after integrating the latter integral by parts. Relation (15) follows from (14) (with $p$ replaced by $p-1$ ) and (13). Note that (15) is a recursion with respect to $q$ only, whereas (13) and (14) are mixed recursions.

\subsection{Series expansion of $\tilde{F}_{q, p}$}

If we substitute the expansion

$$
e^{-a s /(1+s)}=e^{-a} \sum_{k=0}^{x} \frac{1}{k !}\left(\frac{a}{1+s}\right)^{k}
$$

in (11), we obtain

$$
\mathscr{F}_{q, p}=e^{-u} \sum_{k=0}^{\infty} \frac{a^{k}}{k !} \phi_{k+q, p},
$$

with

$$
\phi_{q, p}=\int_{0}^{x} e^{-\zeta s} f(s) \mathrm{d} s .
$$

Expansion (17) is convergent for all complex values of $a$ (and $\zeta$ satisfying (12)), and is useful for small or intermediate values of $a$. If we use the same methods as were used 
for obtaining the recursions for $\mathscr{F}_{q, p}$ in (13)-(15), we obtain recursions for the $\phi_{q, p}$ :

$$
\begin{aligned}
& (b-1) \phi_{q, p}=b \phi_{q-1, p}-\phi_{q, p-1}, \\
& \zeta \phi_{q, p}=1-q \phi_{q+1, p}-p b \phi_{q, p+1}, \\
& b \zeta \phi_{q, p}=1+[\zeta(b-1)-b(q+p)] \phi_{q+1, p}+(b-1)(q+1) \phi_{q+2, p} .
\end{aligned}
$$

Again, the final relation is a recursion with respect to $q$ only. Although expansion (17) converges quite fast (when $|a|$ is not too large), its use is limited when no efficient methods are available for computing two successive values of $\phi_{q, p}$ to start the recursion (21).

An alternative asymptotic expansion of $\widetilde{F}_{q, p}$ can be obtained if we introduce the new function

$$
g(s)=e^{-a s /(1+s)} f(s)
$$

and expand $g(s)$ in a series with respect to $s$, i.e., $g(s)=\sum_{k=0}^{\infty} c_{k} s^{k}$. If we substitute the latter expansion into (11), we obtain

$$
\mathscr{F}_{q, p} \sim \sum_{k=0}^{\infty} c_{k} \frac{k !}{\zeta^{k+1}},
$$

as $\zeta \rightarrow \infty, a$ being fixed. The coefficient $c_{0}$ is equal to 1 . Other coefficients can be generated by recurrence relations which follow from the differential equation

$$
(s+1)^{2}(1+b s) \frac{\mathrm{d} g(s)}{\mathrm{d} s}=-\left[a(1+b s)+q(1+s)(1+b s)+p b(1+s)^{2}\right] g(s) .
$$

As a result we obtain

$$
\begin{aligned}
& c_{1}=-(a+q+b p) c_{0}, \\
& 2 c_{2}=-(2+b+a+q+b p) c_{1}-(a b+q+q b+2 p b) c_{0}
\end{aligned}
$$

and

$$
\begin{aligned}
k c_{k}= & -[(k-1)(b+2)+a+q+p b] c_{k-1}-[(k-2)(2 b+1)+ \\
& +a b+q+q b+2 p b] c_{k-2}-[b(k-3)+q b+p b] c_{k-3},
\end{aligned}
$$

for $k \geq 3$.

Next we derive an asymptotic expansion that is more powerful than the previous one, since it allows also large values of $|a|$. If we introduce a new variable

$$
t=\zeta s+a s /(1+s)
$$

we can rewrite expression (11) as

$$
\mathscr{F}_{q, p}=\int_{0}^{x} e^{-t} h(t) \mathrm{d} t,
$$


where

$$
h(t)=f(s) \frac{\mathrm{d} s}{\mathrm{~d} t}=\sum_{k=0}^{x} e_{k} t^{k} .
$$

This yields the expansion

$$
\bar{F}_{q, p} \sim \sum_{k=0}^{\infty} k ! e_{k} .
$$

In order to find the coefficients $e_{k}$ we first need to find the coefficients of the other expansion

$$
f(s)=\sum_{k=0}^{\infty} f_{k} s^{k}
$$

The coefficient $f_{0}$ is obviously equal to 1 . Other coefficients can be obtained from the differential equation

$$
(1+s)(1+b s) \frac{\mathrm{d} f(s)}{\mathrm{d} s}=-[q+b p+b(p+q) s] f(s) .
$$

As a result we obtain

$$
\begin{aligned}
& f_{1}=-q-p b, \\
& f_{2}=-\left[(b+1+q+p q) f_{1}+b(p+q)\right] / 2
\end{aligned}
$$

and

$$
f_{k}=-\left\{b(k-2+p+q) f_{k-2}+[q+p b+(k-1)(b+1)] f_{k-1}\right\} / k
$$

for $k \geq 3$.

If we use this expansion of $f(s)$ and expand

$$
s=\frac{t-z+\sqrt{(t-z)^{2}+4 t \zeta}}{2 \zeta}
$$

in powers of $t / z$,

$$
s=\frac{t}{z}+\frac{t^{2}}{z^{2} \mu}-\frac{(\mu-2) t^{3}}{z^{3} \mu^{2}}+\frac{\left(\mu^{2}-5 \mu+5\right) t^{4}}{z^{4} \mu^{3}}-\frac{(\mu-2)\left(\mu^{2}-7 \mu+7\right) t^{5}}{z^{5} \mu^{4}}+O\left(t^{6}\right),
$$

we obtain the following expressions for the coefficients $e_{k}$ :

$$
\begin{aligned}
& e_{0}=\frac{1}{z}, \quad e_{1}=\frac{2+\mu f_{1}}{\mu z^{2}}, \quad e_{2}=\frac{6+3 \mu\left(f_{1}-1\right)+\mu^{2} f_{2}}{\mu^{2} z^{3}}, \\
& e_{3}=\frac{20+\mu\left(-20+10 f_{1}\right)+4 \mu^{2}\left(1-f_{1}+f_{2}\right)+\mu^{3} f_{3}}{\mu^{3} z^{4}},
\end{aligned}
$$




$$
e_{4}=\frac{70+35 \mu\left(f_{1}-3\right)+15 \mu^{2}\left(f_{2}-2 f_{1}+3\right)+5 \mu^{3}\left(f_{3}-f_{2}+f_{1}-1\right)+\mu^{4} f_{4}}{\mu^{4} z^{5}},
$$

with $\mu=z / a$. Expansion (25) holds for large values of $|z|$ and $|a|$, as long as $\mu$ is not close to zero. Complex values of $z$ and $a$ are allowed in the range of physical interest:

$$
\arg z \in(-\pi, \pi], \quad \Re a>0 .
$$

Expansion (23) is valid only for fixed values of $a$, and large values of $|z|$, and so its use is limited, compared with expansion (25). However, the coefficient $c_{k}$ can be evaluated by using recursion (24), which is much simpler than the expressions for the coefficients $e_{k}$.

\section{Approximations to $\overline{\mathscr{F}}_{q, p}$}

In this section we summarize (after some rearrangement) some approximations to $\pi_{4}, p$ which were obtained by ST for $a \gg 1$ (in this section $a$ and $z$ are assumed to be real) and which will be later, in Section 5, compared with the results of a numerical analysis of $\tilde{F}_{q, p}$ based on the numerical code described in Section 4.

In the limit $a \gg 1$ and $z<a$, we have the following approximations for $\Re \mathscr{F}_{q, p}$ and $\mathfrak{F} \bar{F}_{q, p}$.

$$
\begin{aligned}
& \Re \mathscr{\mathscr { F }}_{q, p} \sim \Re \mathscr{F}_{q, p}^{A} \equiv \frac{1}{2 \sqrt{a}}\left\{-\Re Z-\frac{\xi}{2 \sqrt{a}}\left(\Re Z+\xi \Re Z^{\prime}\right)+\frac{1-q-p b}{2 \sqrt{a}} \Re Z^{\prime}\right\}, \\
& \mathfrak{F}_{q, p} \sim \mathscr{F}_{4} \cdot \mathscr{F}_{q, p}^{A} \equiv-\frac{\sqrt{\pi}(1-\mu)^{(q+p-1.5) / 2}}{2 \sqrt{a}[b+(1-b) \sqrt{1-\mu}]^{p}} e^{-a x_{i)}^{2}},
\end{aligned}
$$

where $\mu=z / a, x_{0}=\sqrt{2-\mu-2 \sqrt{1-\mu}}, \xi=z /(2 \sqrt{a}), Z \equiv Z(\xi)$ is the non-relativistic plasma dispersion function determined by the equation

$$
\begin{aligned}
& Z(\xi)=i \sqrt{\pi} \exp \left(-\xi^{2}\right)-2 \int_{0}^{\xi} \exp \left(-\xi^{2}+t^{2}\right) \mathrm{d} t \\
& Z^{\prime}=-2(1+\xi Z(\xi)) .
\end{aligned}
$$

Sazhin and Temme (1991) have recently generalized expression (27) to include the second term in the expansion with respect to $a^{-1}$. However, for $|a| \gg 1$ this term, being of the order of $a^{-3 / 2}$, could change the value of $\& \pi_{q, p}$ only slightly and it will not be considered in this paper.

In the limiting case $\xi \rightarrow-\infty$, but retaining the first-order terms, we simplify $\mathscr{F}_{q, p}$ to

$$
\Re, \mathbb{F}_{q, p} \sim \Re, \mathbb{F}_{q, p}^{B} \equiv \frac{1}{z}+\frac{a}{z^{3}}\left(2-\frac{(q+b p) z}{a}\right) .
$$


In the non-relativistic case $a \rightarrow \infty$ the expression (26) reduces to

$$
\Re \mathscr{F}_{q, p} \sim \Re \mathscr{F}_{q, p}^{C} \equiv-\frac{1}{2 \sqrt{a}} \mathfrak{R} Z\left(\frac{z}{2 \sqrt{a}}\right) .
$$

The expression (27) can be simplified in the limit $|\mu| \ll 1$ and $|\mu| \xi^{2} / 2 \ll 1$ and reduced to

$$
\mathfrak{F}_{q, p} \sim \mathscr{F}_{q, p} \mathscr{F}_{q, p}^{B} \equiv-\frac{i \sqrt{\pi}}{2 \sqrt{a}}\left[1+\frac{\xi}{2 \sqrt{a}}\left(3-2 \xi^{2}-2 q-2 b p\right)\right] \exp \left(-\xi^{2}\right) .
$$

In the non-relativistic case $a \rightarrow \infty$ the expression (32) is simplified to

$$
\mathfrak{F} \mathscr{F}_{q, p} \sim \mathcal{F}_{\mathscr{F}^{C}, p}^{C} \equiv-\frac{i \sqrt{\pi}}{2 \sqrt{a}} \exp \left(-\xi^{2}\right) .
$$

All the approximations for $\mathscr{F}_{q, p}$ given in this section are based on asymptotic analyses of $\pi_{q, p}$. We believe that the best way to specify rigorously the range of applicability of these approximations is to compare them with the results of numerical analysis of $\pi_{q, p}$. This will be done in Section 5 after we have summarized the basic principles of the numerical evaluation of $\tilde{F}_{q, p}$ in the next section.

\section{Numerical Evaluation of $\mathscr{F}_{q, p}$}

Expansion (25) is useful when the parameters $|a|$ and $|z|$ are large under the condition that both $\mu=z / a$ and $\mu z$ are bounded away from zero. It is not possible, however, to use this expansion for the computation of the imaginary part of $\mathscr{F}_{q, p}$ when $\zeta=z-a$ is negative (with $a$ and $z$ real). Thus we cannot use it for the analysis of whistler-mode growth or damping in a weakly relativistic plasma. In this case we should use either the expansions of ST discussed in the previous sections, or the numerical code described below. This will be based on quadrature of the integral defining $\mathscr{F}_{q, p}$. This method will be useful for small or moderate values of both parameters $|a|$ and $|z|$, for which range efficient series expansions are not available, or when $|a|$ is large with respect to $|z|$. We have used the NAG-routine D01AKF.

When $a$ and $z$ are complex, we have to deform the path of integration of (11) in order to obtain the proper analytic continuation of $\mathscr{F}_{q, p}$. Another point is that for large values of $|a|$ and $|z|$ the integrand may oscillate very rapidly, and so we should choose the contour of integration in such a way as to avoid these oscillations whenever possible.

When $|a|$ is large with respect to $|z|$, it is possible to define a simple contour of integration, on which an efficient quadrature rule can be based. We write (11) in the form

$$
\mathscr{F}_{q, p}=\int_{0}^{\infty} e^{-z s+a s^{2} /(1+s)} f(s) \mathrm{d} s,
$$


and concentrate on the term $\phi(s):=a s^{2} /(1+s)$ of the exponential function. We try to find a contour on which the imaginary part of $\phi(s)$ is constant; since the contour should start as $s=0$, and since $\phi(0)=0$, this constant must be zero. Let

$$
\begin{array}{ll}
a=\beta e^{i \alpha}, \quad\left(|\alpha|<\frac{1}{2} \pi\right), \quad \zeta=z-a=\gamma e^{i \delta}, \quad(|\delta| \leq \pi), \\
s=\sigma e^{i \tau}, \quad(|\tau|<\pi) .
\end{array}
$$

Then the path through the origin satisfying $₹ \phi(s)=0$ is defined by

$$
\mathscr{F}\left[\frac{e^{i(x+2 \tau)}}{1+\sigma e^{i \tau}}\right]=0,
$$

from which we can solve for $\sigma$ :

$$
\sigma=-\frac{\sin (\alpha+2 \tau)}{\sin (\alpha+\tau)} .
$$

Thus the path in the $s$-plane can be described by $\tau$, which gives $\sigma(\tau)$ and we have to determine an appropriate interval of $\tau$. The initial value $\tau_{0}$ of $\tau$ should satisfy $\sigma\left(\tau_{0}\right)=0$. Hence, $\tau_{0}$ satisfies $\alpha+2 \tau_{0}=k \pi$. Similarly, the final value $\tau_{f}$ is given by $\alpha+\tau_{f}=l \pi$. The integer values $k$ and $l$ have to be determined.

Two conditions for selecting $k$ and $l$ should be considered: convergence of the integral at infinity and taking the proper analytic branch of the many-valued function $\mathscr{F}_{q, p}$. The choice $k=0$, giving $\tau_{0}=-\alpha / 2$, gives a divergent integral, since we start in the wrong (uphill) direction at the saddle point $s=0$ of $\exp (\phi(s))$. According to the remarks on analytic continuation in Section 2.1, we should start and stay in the upper (lower) plane when $\zeta$ is in the lower (upper) plane. This gives the value $k=l=-1 \cdot \operatorname{sign}(\delta)$, and

$$
\tau_{0}=-\operatorname{sign}(\delta) \pi / 2-\alpha / 2, \quad \tau_{f}=-\operatorname{sign}(\delta) \pi-\alpha .
$$

In other words, $\tau_{f}=2 \tau_{0}$. It follows that the starting direction for the integration of $\tilde{F}_{q, p}$ is chosen in the sector $\pi / 4<\arg s<3 \pi / 4$ when $\zeta$ is in the lower half-plane, and $-3 \pi / 4<\arg s<-\pi / 4$ when $\zeta$ is in the upper half-plane.

However, the absolute value of $\tau_{f}$ may be larger than $\pi$, and values of $|\tau|$ larger than $\pi$ should be avoided, due to the branch lines of the many-valued function $f(s)$ in (7) and (8). We may modify this value $\tau_{f}$, however. The exact value is not important, since the exponential function soon becomes small along the path of integration. When $\left|\tau_{f}\right|>\pi$ we may replace it with $-\operatorname{sign}(\delta) \pi$, and in that case we replace the above definition of $\sigma$ with $\sigma=-\sin (\alpha+2 \tau) / \sin (\tau)$. In this way we have $\sigma\left(\tau_{f}\right)=+\infty$.

To summarize, in the computer program we integrate with respect to $\tau \in\left[\tau_{0}, \tau_{f}\right]$, and we use the path of integration given by

$$
\sigma=-\frac{\sin (\alpha+2 \tau)}{\sin \left(\alpha_{0}+\tau\right)}, \quad \tau \in\left[\tau_{0}, \tau_{\mathrm{f}}\right)
$$


where $\tau_{0}=-\operatorname{sign}(\dot{\delta}) \pi / 2-\alpha / 2$ and

$$
\begin{array}{ll}
\alpha_{0}=\alpha, \quad \tau_{f}=-\operatorname{sign}(\delta) \pi-\alpha, & \text { if } \quad \operatorname{sign}(\delta) \neq \operatorname{sign}(\alpha) ; \\
\alpha_{0}=0, \quad \tau_{f}=-\operatorname{sign}(\delta) \pi, \quad \text { if } \quad \operatorname{sign}(\delta)=\operatorname{sign}(\alpha) .
\end{array}
$$

This numerical method is the basis of the investigations of the next section.

\section{Comparison between Numerical and Analytical Results}

We restrict our comparison between numerical results and the results of the asymptotic analyses shown in Section 3 to real values of $a$ and $z$ only, as the asymptotical results were derived for real $a$ and $z$. This restriction is not very serious for the application to whistler-mode waves, as for most practically important cases of whistler-mode propagation in magnetospheric plasma the following inequality is valid:

$$
\mathscr{F} \omega \ll \min \left(\Re \omega, \Omega_{0}-\mathfrak{R} \omega\right),
$$

where $₹ \omega$ is the increment of whistler-mode instability or the decrement of damping. In this case the analysis of the propagation of these waves and their instability or damping reduces to the analysis of $\mathfrak{R} \mathscr{F}_{q, p}$ and $\mathscr{F}_{\mathcal{F}_{, p}}$ for real values of their arguments (see, e.g., ST).

Remembering the definition of $a$ and the fact that $r \ll 1$ in the magnetosphere and $N>1$ for whistler-mode waves, we can anticipate that the condition $a \gg 1$, used for the asymptotic analysis in Section 3, is satisfied.

Also, we restrict our comparison to $q=0.5$ and 1.5 and $p=2$ as these $q$ and $p$ are actually used in Equation (1). We consider two values of $b: b=1$ and $b=2$ which corresponds to $A_{e}=1$ and $A_{e}=2$. Most of the actual values of $A_{e}$ observed in the magnetosphere lie between these, i.e., $1<A_{e}<2$. As to the value of $a$ we first assume it to be equal to 100 .

Numerical plots of $\Re \cdot \mathscr{F}_{1 / 2,2}$ as well as plots of $\Re \mathscr{F}_{1 / 2,2}^{A} \mathfrak{R} \cdot \tilde{F}_{1 / 2,2}^{B}$ and $\Re \mathscr{F}_{1 / 2,2}$ for $b=1$ are shown in Figure 1(a) for $-90 \leq \mathfrak{R} z \leq 0$. For $|\mathfrak{R} z| \gtrsim 90$ we have $|\xi| \gg 1$ and the asymptotic expression (30) can be used.

As follows from Figure 1(a), $\Re \mathscr{H}_{1 / 2,2}^{A}$ almost coincides with numerical values of $\Re, \widetilde{F}_{1 / 2,2}$, to within the accuracy of plotting for all $\Re z$ under consideration. This suggests that both the expression (26) obtained by ST and our numerical code are valid. For $\mathfrak{R} z$ approaching - 90, $\Re, \mathscr{F}_{1 / 2,2}^{B}$ becomes a good approximation as well. In any case $\Re, \mathscr{F}_{1 / 2,2}^{B}$ is a better approximation than the non-relativistic approximation $\Re, \bar{F}_{1 / 2,2}$ when $R z \gtrsim 50$. However, if a high accuracy of approximation is not required then $\Re \cdot \bar{H}_{1 / 2,2}$ can be used for $\Re z$ under consideration except when $\Re z$ is very close to zero.

In Figure 1(b) we show the same curves as in Figure 1(a) but for $\mathbb{F}_{1 / 2,2}$ and its

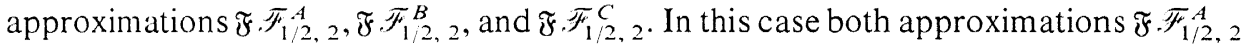
and $\mathscr{F} \cdot \tilde{H}_{1 / 2,2}^{B}$ coincide with the numerical values of $\mathfrak{F} \cdot \overline{\mathscr{F}}_{1 / 2,2}$, to within the accuracy of plotting in the whole range of $\Re z$ under consideration. Also, approximation $\mathfrak{F}_{1 / 2,2} \tilde{F}_{1 / 2}$ is of sufficient accuracy for qualitative analysis. 
Figures 2(a) and 2(b) show curves similar to those in Figures 1(a) and 1(b) but for $b=2$. The general features of the curves in Figures 2(a) and 2(b) are essentially the same as in Figures 1(a) and 1(b) although the difference between the numerical and asymptotical curves is greater than in the case shown in Figures 1(a) and 1(b).

Figures 3(a), 3(b), 4(a), and 4(b) show curves similar to those in Figures 1(a), 1(b), 2(a), and 2(b) but for $\mathscr{F}_{3 / 2,2}$ and its approximations. The general shapes of the curves in Figures 3(a), 3(b), 4(a), and 4(b) are similar to those in Figures 1(a), 1(b), 2(a), and 2(b). This means that the values of $\tilde{F}_{q, 2}$ and its approximations are not very sensitive to the value of $q$ in the range $\frac{1}{2}<q<\frac{3}{2}$.

Note that the values of $\Re \mathscr{F}_{1 / 2,2}, \Re \mathscr{F}_{1 / 2,2}^{A}, \Re \mathscr{F}_{3 / 2,2}, \Re \mathscr{F}_{3 / 2,2}^{A}$ for $a=100$ and at $\Re z$ close to zero, become positive. However, in this case the condition (34) becomes invalid, and so the comparison of the curves in this range has no practical significance. This comparison will be shown later for $a=30$ and $a=10$.

In Figures 5(a), 5(b), 6(a), 6(b), 7(a), 7(b), 8(a), and 8(b) we show curves similar to those in Figures 1(a), 1(b), 2(a), 2(b), 3(a), 3(b), 4(a), and 4(b) but for $a=30$. In this case we considered $\Re z$ in the range $-30 \leq \Re z \leq 0$. For $|\Re z|>30$, we have $|\xi| \gg 1$ and expression (3) can be used as in the case shown in Figures 1-4.

As in Figures 1-4 the numerical curves for $a=30$ appear to be closest to the approximations $\Re \mathscr{F}_{q, p}^{A}$ and $\mathscr{F}_{\mathcal{F}^{\prime}, p}{ }^{A}$ although the differences between the numerical results and the approximation $\Re_{\mathcal{F}^{\prime}}{ }^{A}$ are quite substantial, especially for $q=1.5$ and $b=2$. The approximation $\Re \mathscr{F}_{q, p}^{B}$ is better than the non-relativistic approximation $\Re \mathscr{F}_{q, p}^{C}$ only at $\Re z$ close to $a(\mathfrak{R} z \gtrsim 20)$. The approximation $\mathfrak{F}_{\mathbb{F}_{q}}^{B}$, is better than the approximation $\mathfrak{F}_{\mathscr{F}, p} \bar{F}_{q}$ for all $\Re z$ under consideration.

In Figures 9 to 12 we show curves similar to those shown in Figures 1 to 8 but for $a=10$. The curves for $\Re, \widetilde{F}_{1 / 2,2}$ and $\Re \mathscr{F}_{3 / 2,2}$ and their approximations are similar to those shown in Figures 4(a) to 8(a), although the differences between the curves are slightly greater. Similar agreement is observed for $\widetilde{F}_{1 / 2,2}$ and $\mathbb{F}, \widetilde{F}_{3 / 2,2}$ and their approximations for $b=1$. However, all approximations for $\widetilde{F}_{1 / 2,2}$ and $\widetilde{F}_{3 / 2,2}$ especially $\mathfrak{F}_{1 / 2,2}$

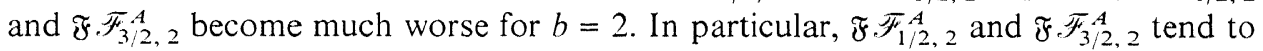
infinity as $\Re z$ approaches $a-(b /(b-1))^{2} \approx-30$ for $b=2$ and $a=10$ which is consistent with Figures 10(b) and 12(b). Hence, one should be cautious when using the approximations discussed in Section 3 even if the condition $a \gg 1$ is valid.

For $a=3$ the difference between the numerical plots $\Re \mathscr{F}_{1 / 2,2}, \mathfrak{R} \cdot \mathscr{F}_{3 / 2,2}, \mathbb{F}_{1 / 2,2}$ and $F \pi_{3 / 2,2}$, and their approximations becomes even greater. However, this case is of little importance for magnetospheric applications and it will not be discussed here.

\section{Principal Conclusions}

(a) $\mathfrak{R} \mathscr{F}_{4, p}^{A}$ and $\mathfrak{F} \cdot \mathscr{F}_{q, p}^{A}$ (see Equations (26) and (27)) seem to be almost ideal approximations for the functions $\Re, \mathbb{F}_{q, p}$ and $\mathfrak{F} . \mathbb{F}_{q, p}$ for $\mathfrak{R} z \gtrsim 100, \mathfrak{F} a=\mathfrak{F} z=0, q=\frac{1}{2}, \frac{3}{2} ; p=2$ and $b=1 ; 2$ (these values of $q, p$, and $b$ will be also assumed in other paragraphs in this section) over a wide range of $\Re z$ provided the condition (34) is valid.

(b) $\Re \cdot \mathscr{F}_{q, p}^{B}$ (see Equation (30)) is better than the non-relativistic expression $\Re \mathscr{F}_{q, p}^{C}$ (see 


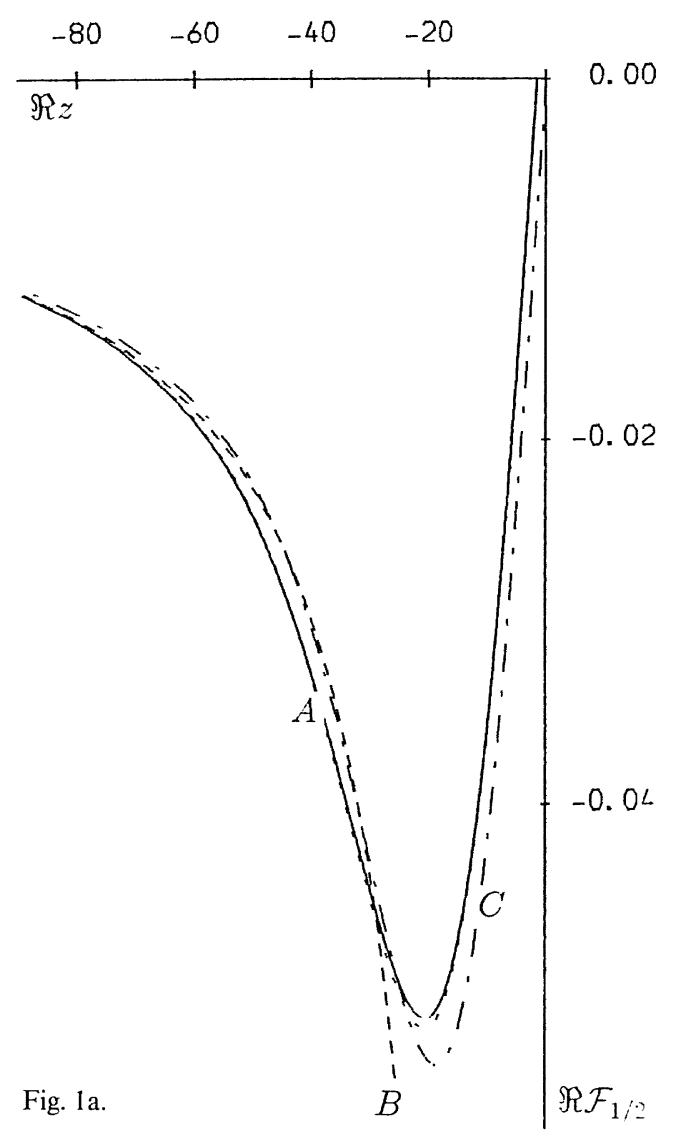

F1g. la. Numerical plots of $\Re \widetilde{\mathscr{F}}_{q, p}$ (Equation (2), solid curve) and the approximations $\Re \mathscr{F}^{A}$ (Equation (26), dashed curve $A$ ), $\Re \mathscr{F}_{a, p}^{B}$ (Equation (30), dashed curve $\mathrm{B}$ ) and $\Re \mathscr{F}_{q, p}^{C_{1}}$ (Equation (31), dashed curve $C$ ) versus $\Re z$ for $\Re a \equiv a=100, q=\frac{1}{2}, p=2$, and $b=1$.

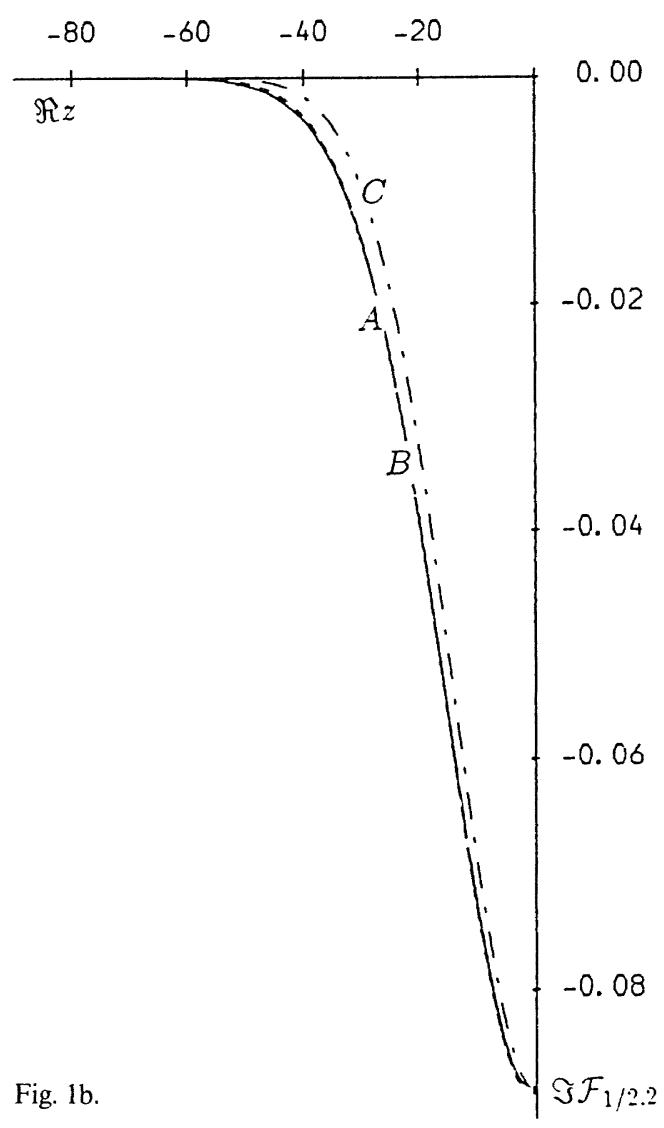

Fig. 1b. The same as Figure 1(a) but for $\mathbb{F}_{\mathscr{q}, p}$ (Equation (2), solid curve) and the approximations $\widetilde{F}_{4, p} \mathscr{F}^{A}$,

(Equation (27), dashed curve $A$ ), $\mathfrak{F}^{\mathscr{F}_{a p}}{ }^{B}$ (Equation (32), dashed curve $B$ ) and $\mathscr{F}_{q, p} \mathscr{F}^{C}$ (Equation (33), dashed curve $C$ ). 


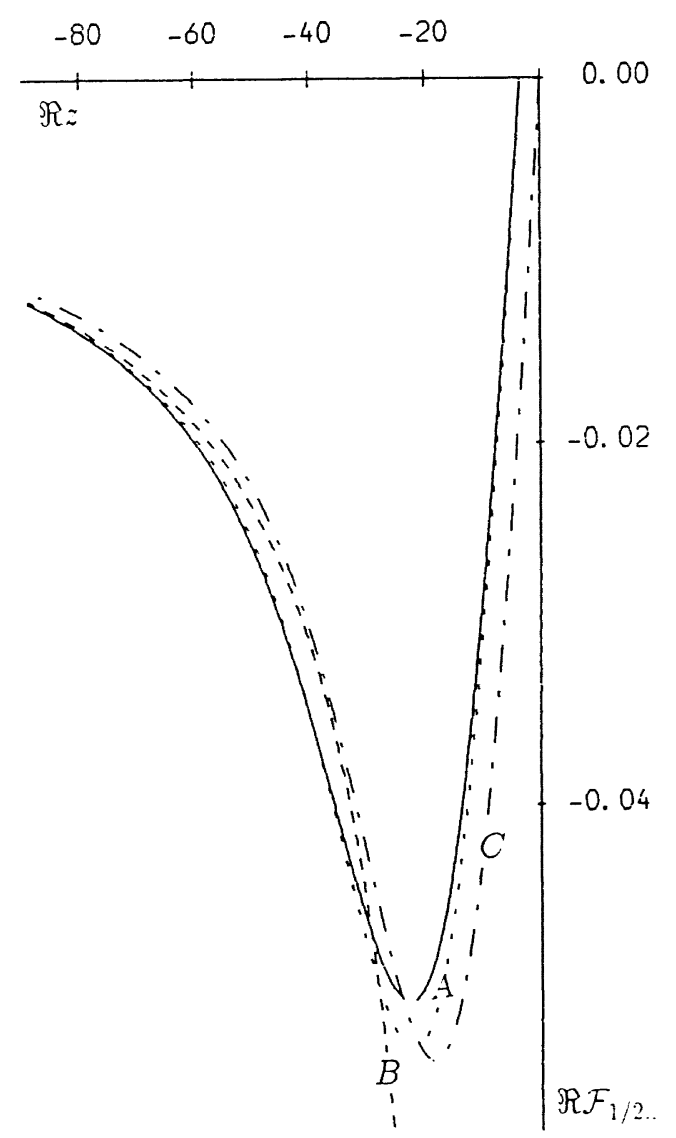

Fig. 2a. The same as Figure 1(a) but for $b=2$.

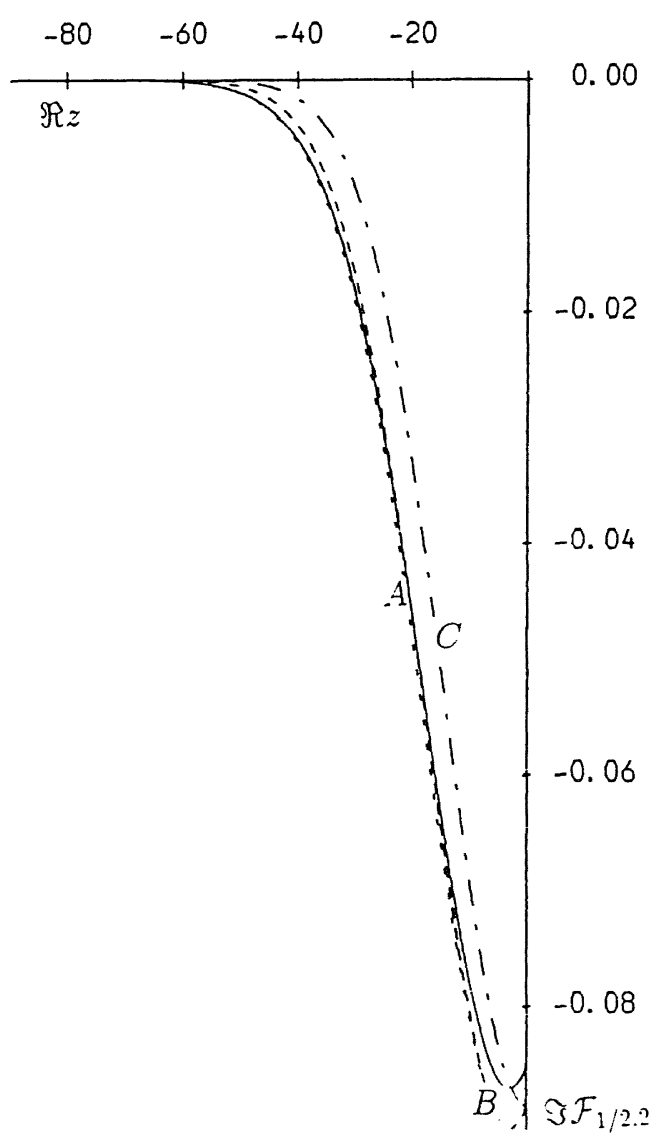

Fig. 2b. The same as Figure 1(b) but for $b=2$. 


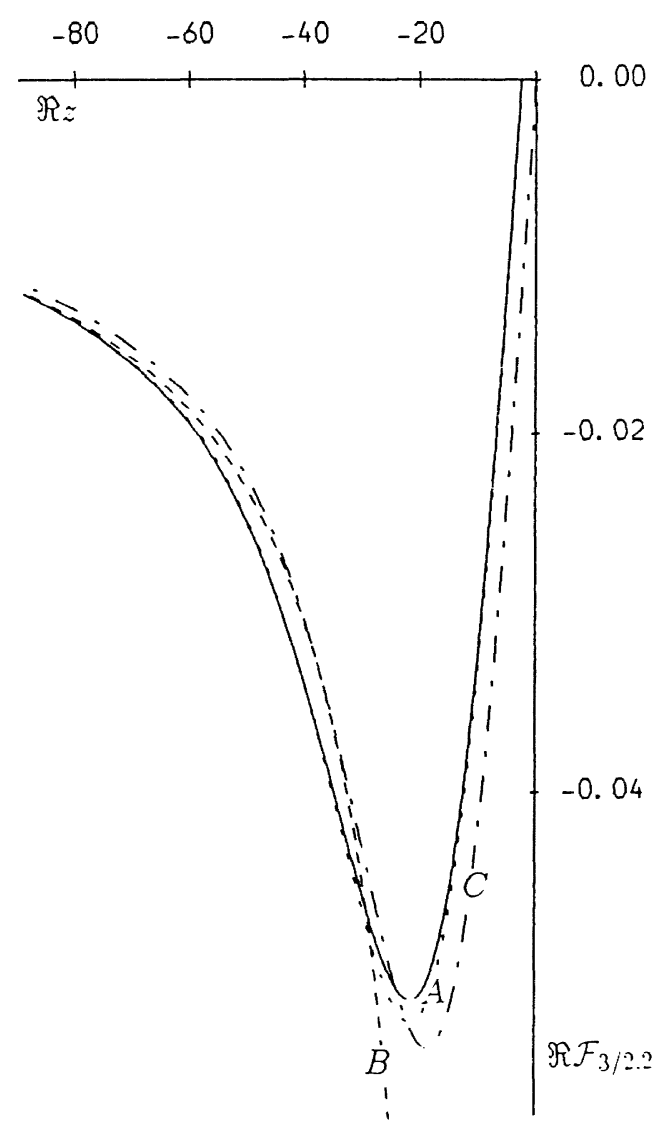

Fig. 3a. The same as Figure 1(a) but for $q=1.5$.

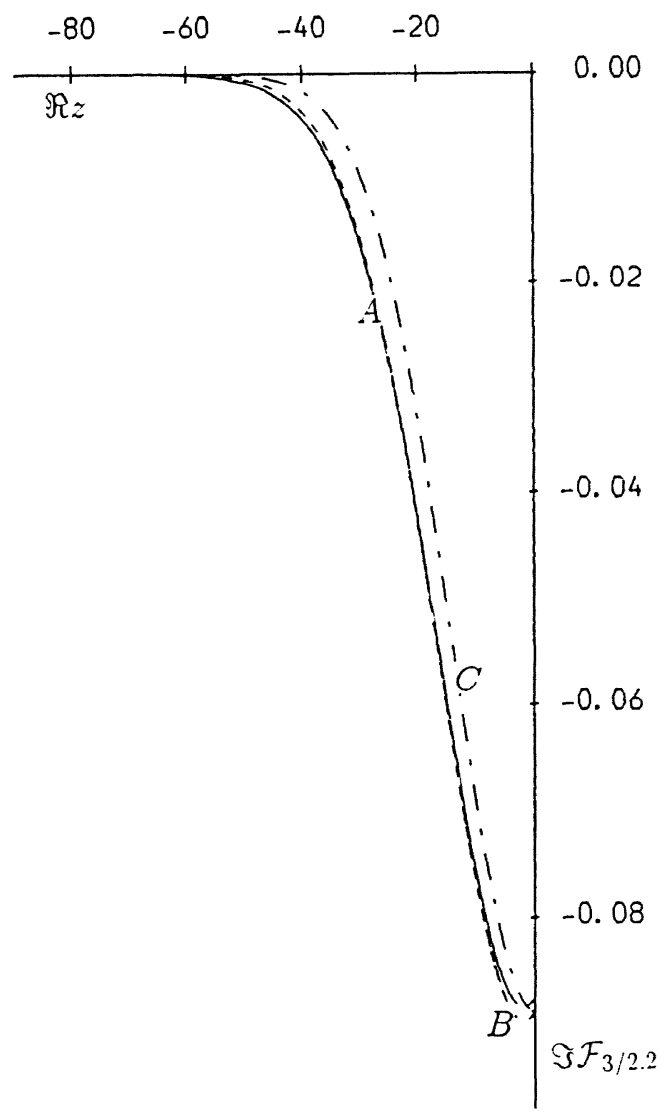

Fig. 3b. The same as Figure 1(b) but for $q=1.5$ 


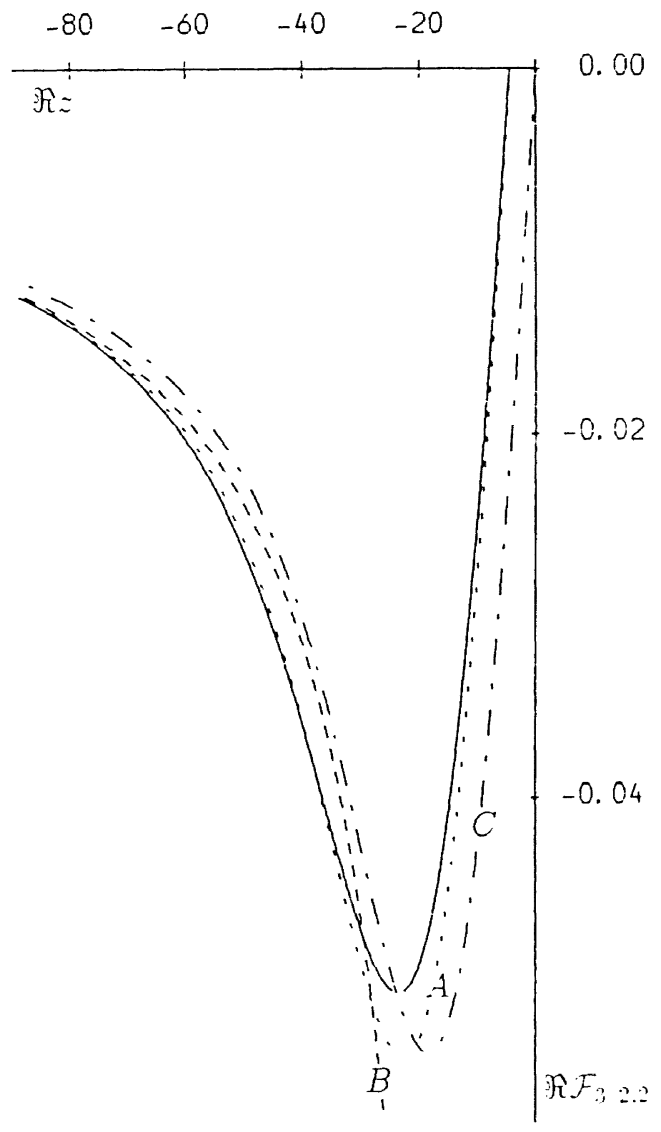

Fig. 4a. The same as Figure 1(a) but for $q=1.5$ and $b=2$.

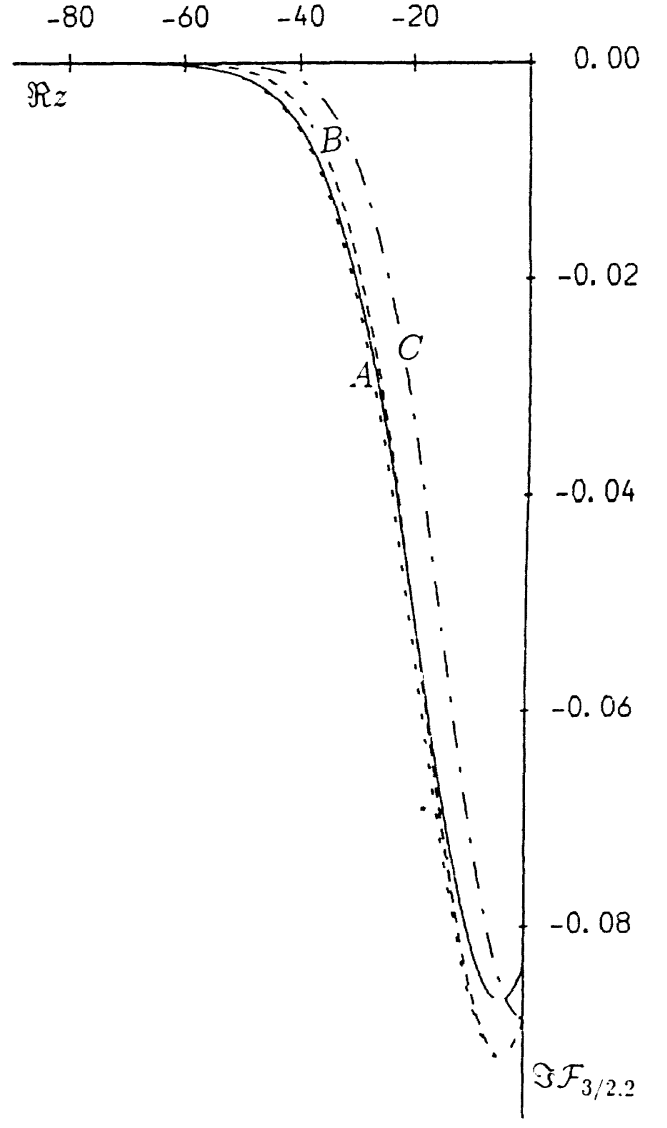

Fig. 4b. The same as Figure 1(b) but for $q=1.5$ and $b=2$. 


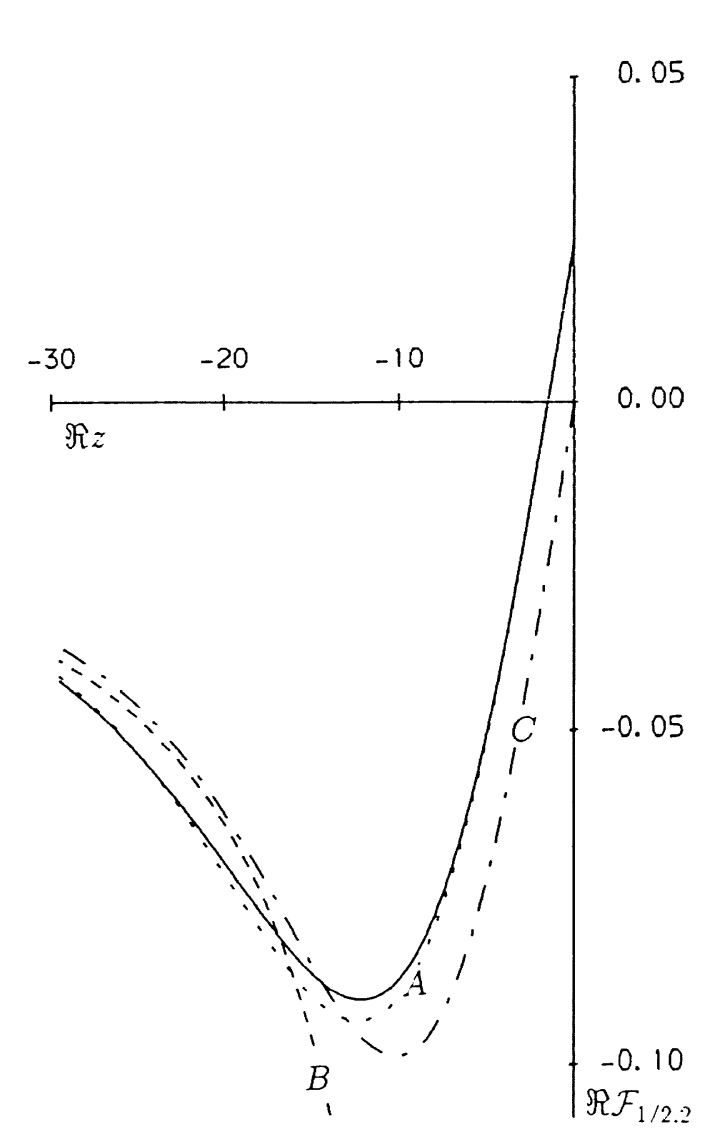

Fig. 5a. The same as Figure 1(a) but for $a=30$.

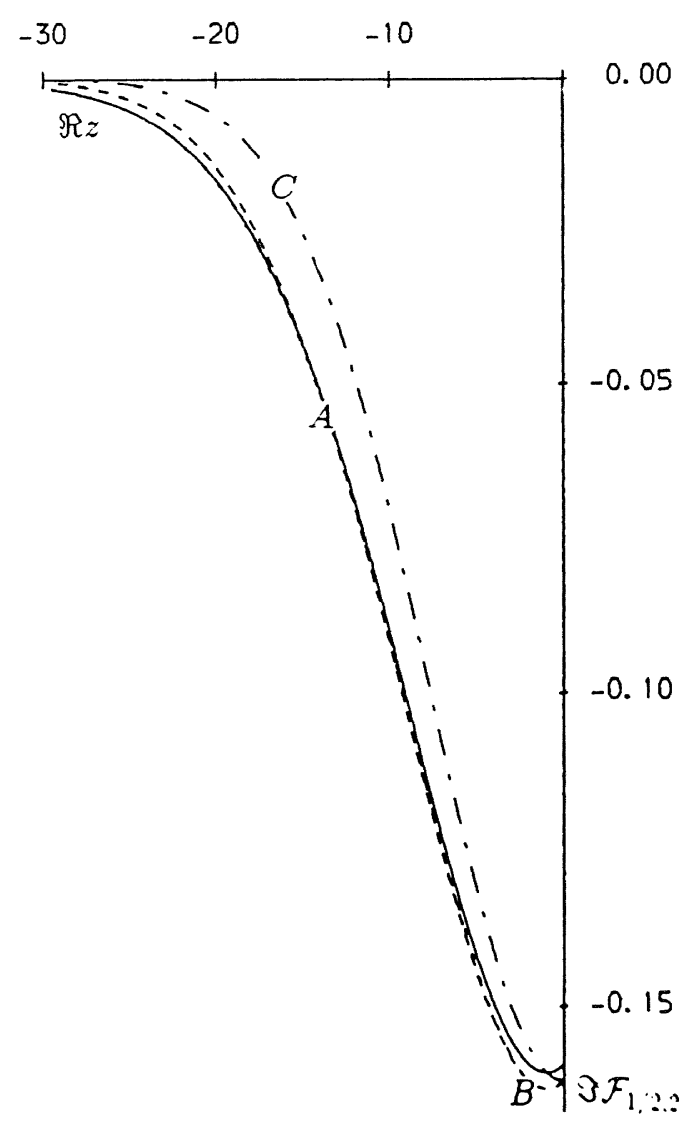

Fig. 5b. The same as Figure 1(b) but for $a=30$. 


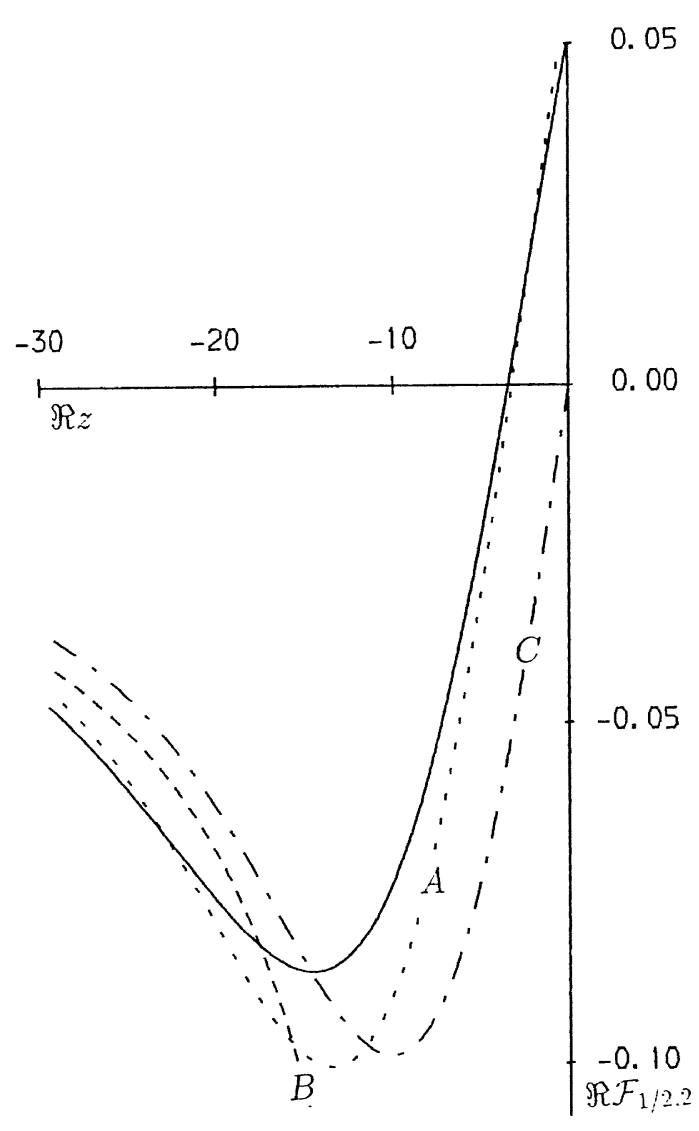

Fig. 6a. The same as Figure 2(a) but for $a=30$.

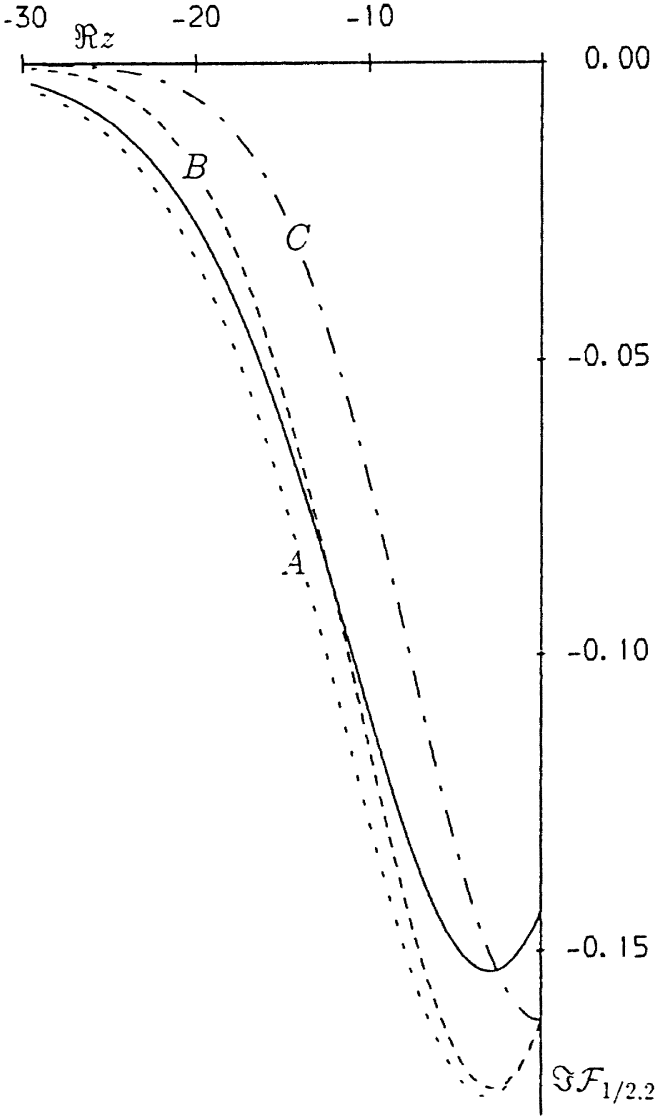

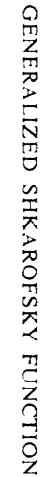

Fig. 6b. The same as Figure 2(b) but for $a=30$. 


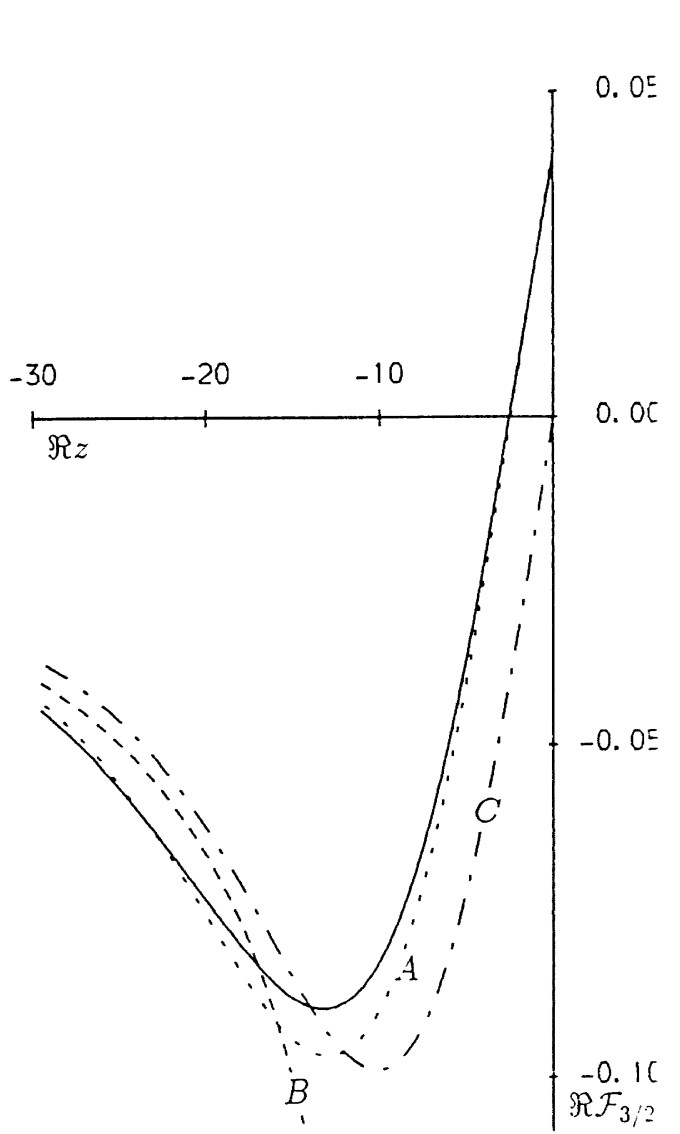

Fig. 7a. The same as Figure 3(a) but for $a=30$.

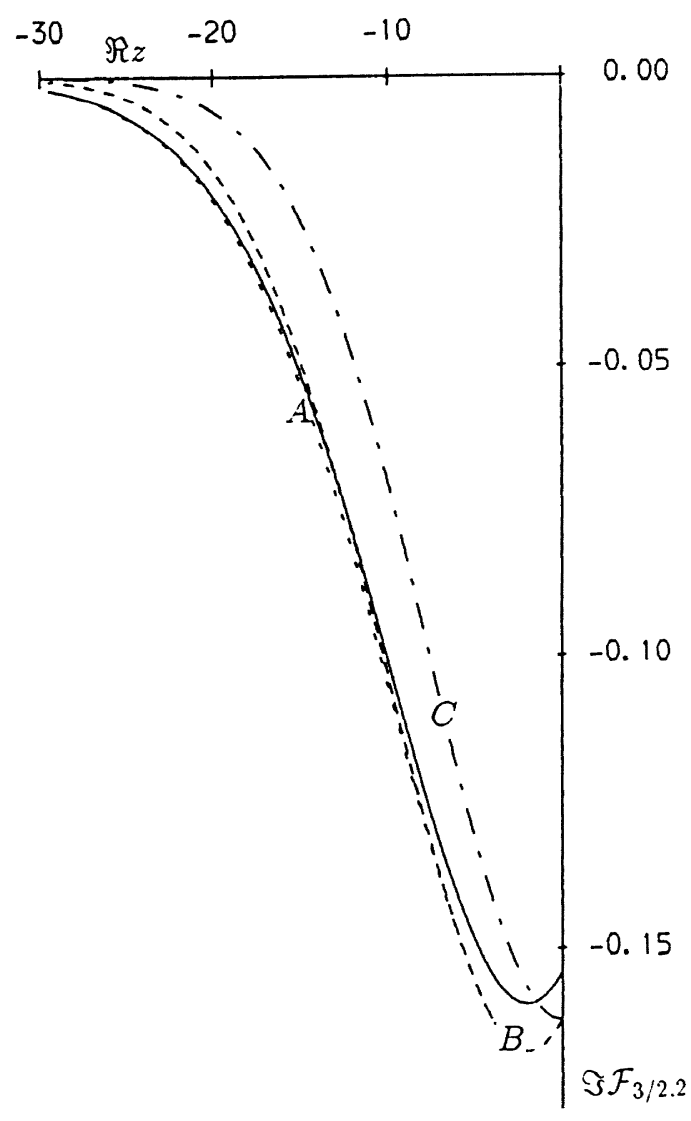

Fig. 7b. The same as Figure 3(b) but for $a=30$. 


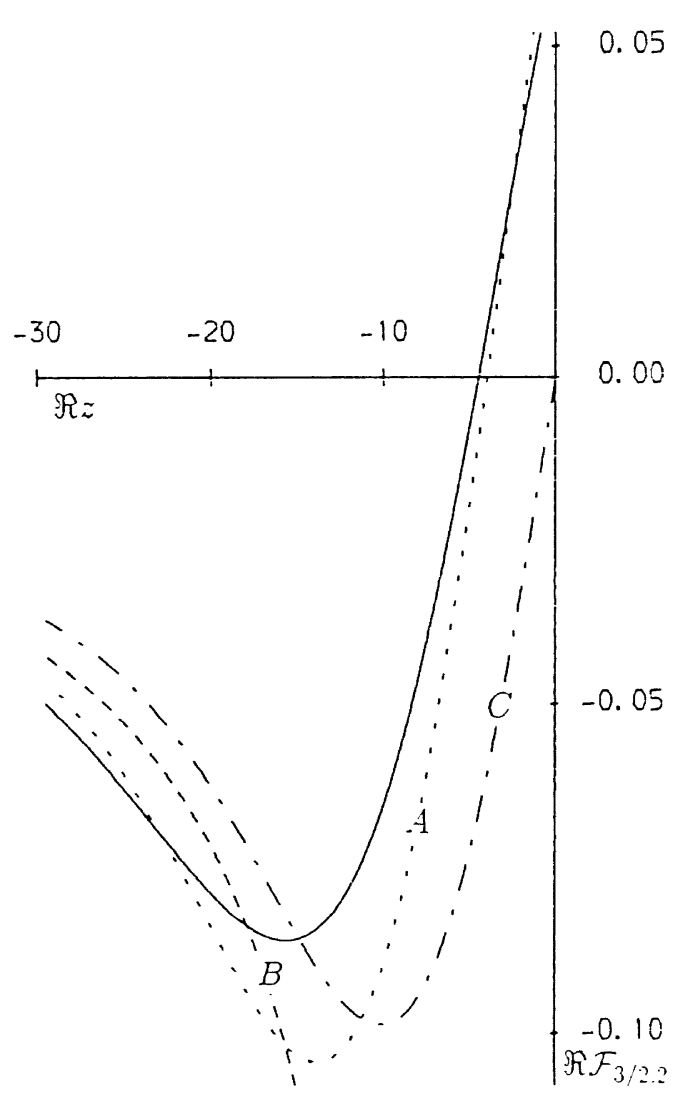

Fig. 8a. The same as Figure 4(a) but for $a=30$

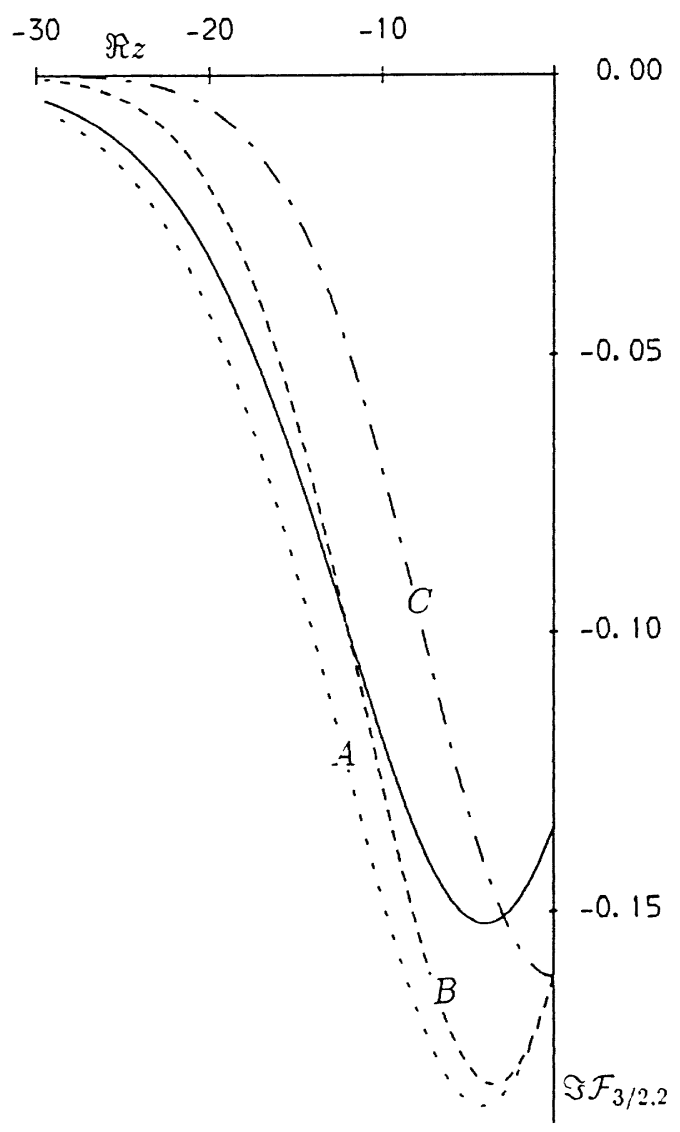

Fig. 8b. The same as Figure 4(b) but for $a=30$. 


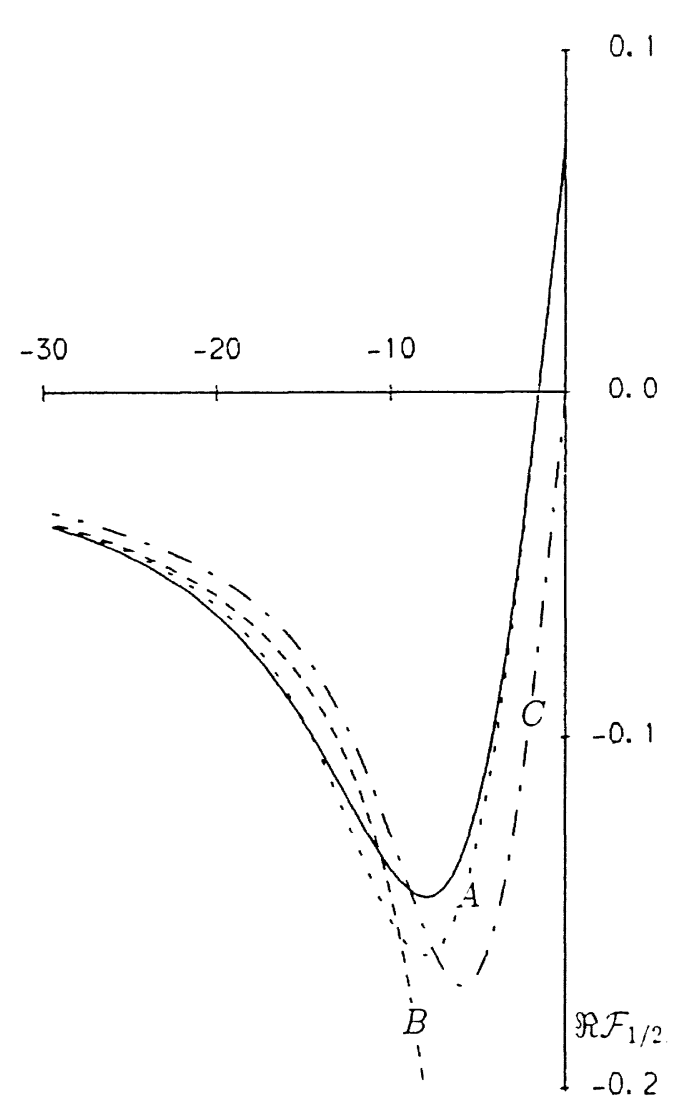

Fig. 9a. The same as Figure 1(a) but for $a=10$.

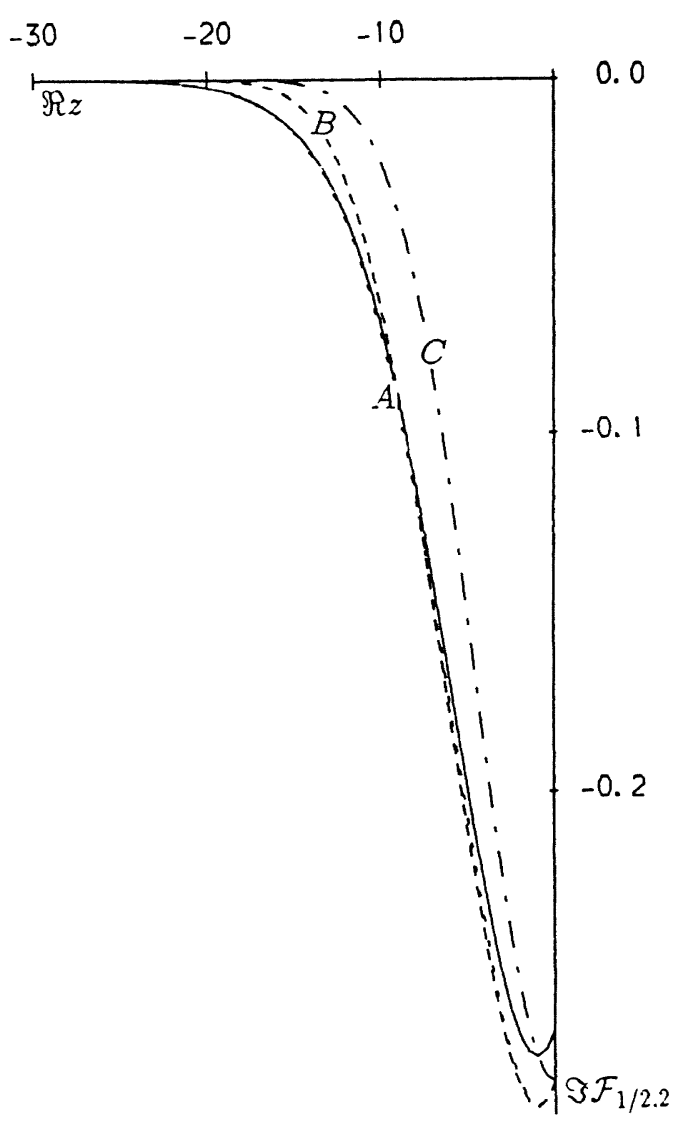

Fig. 9b. The same as Figure 1(b) but for $a=10$. 


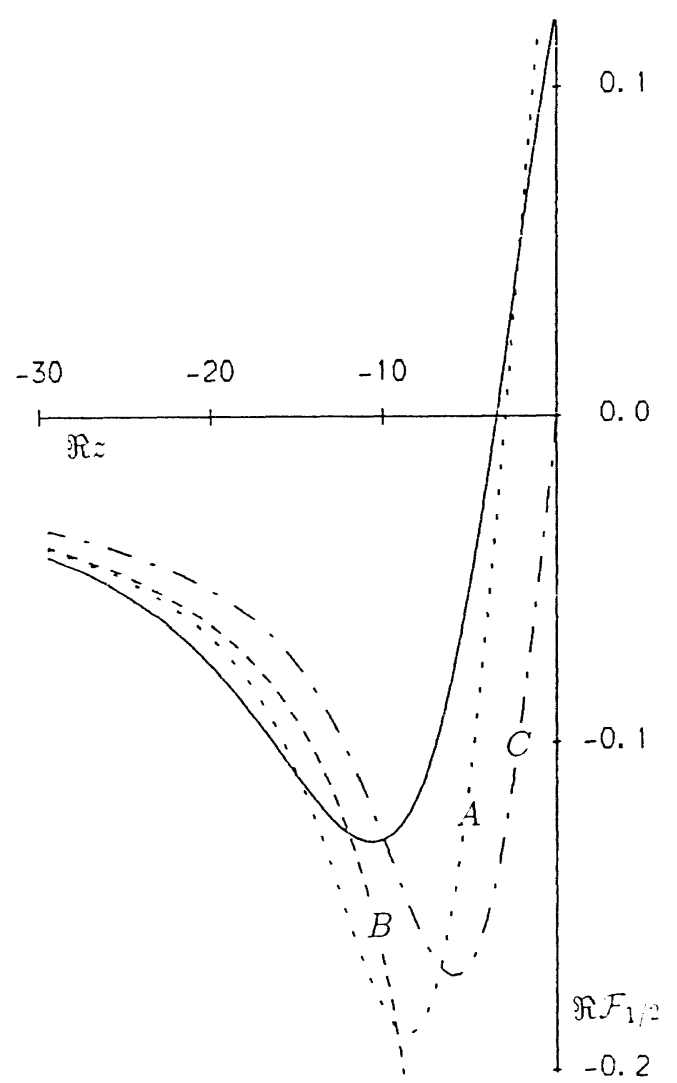

Fig. 10a. The same as Figure 2(a) but for $a=10$.

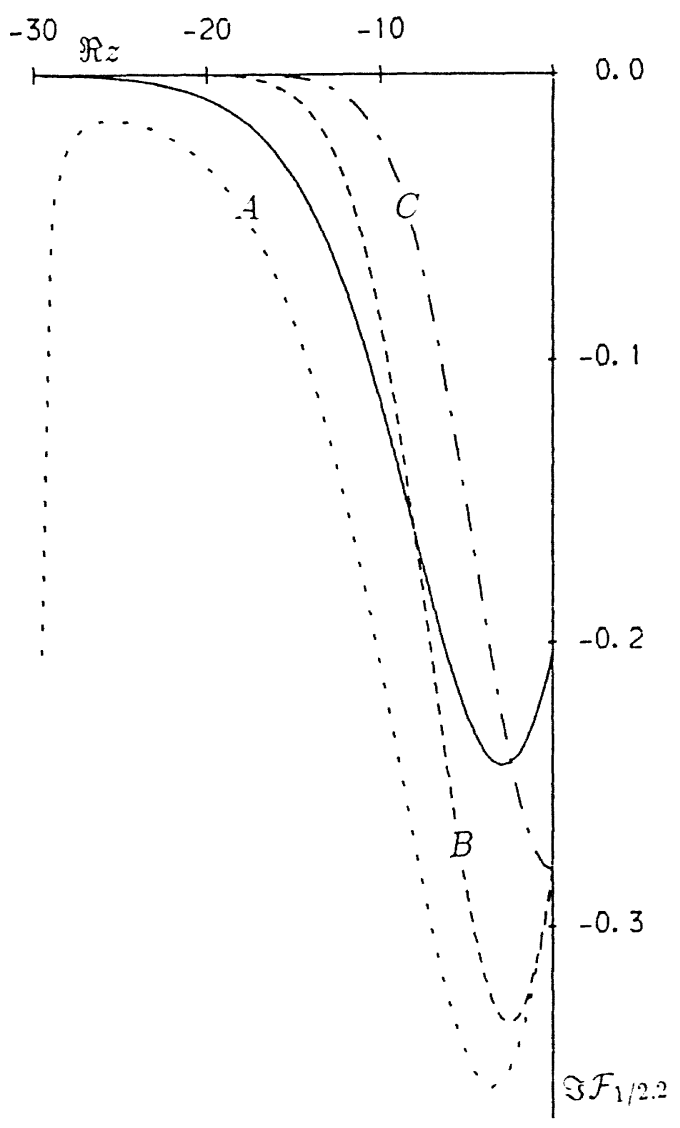

Fig. 10b. The same as Figure 2(b) but for $a=10$. 


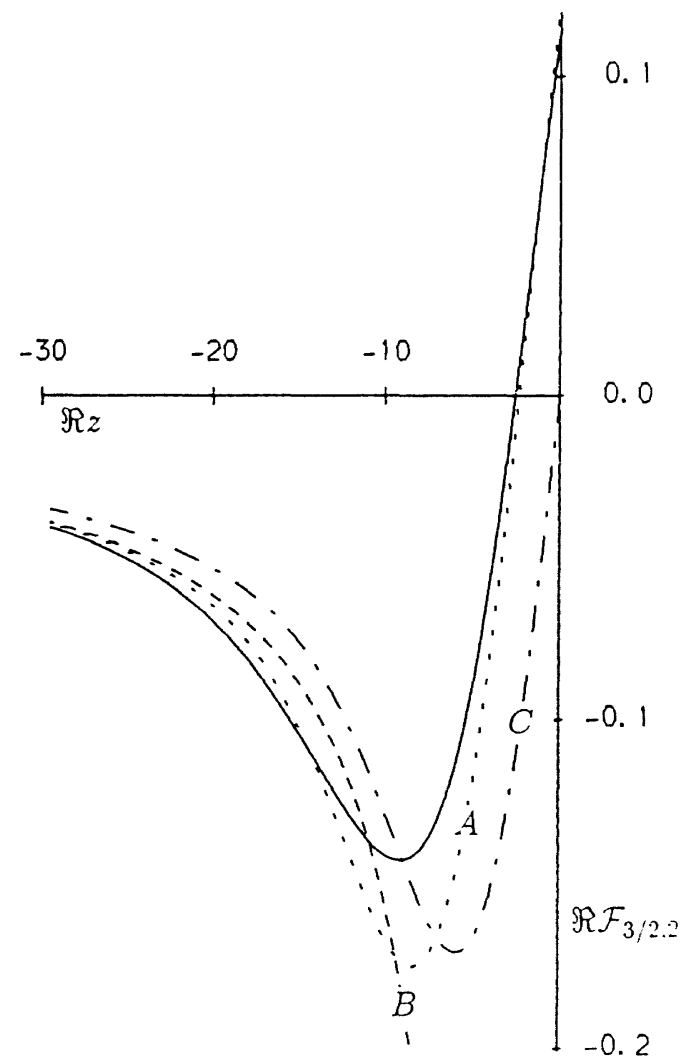

Fig. 11a. The same as Figure 3(a) but for $a=10$.

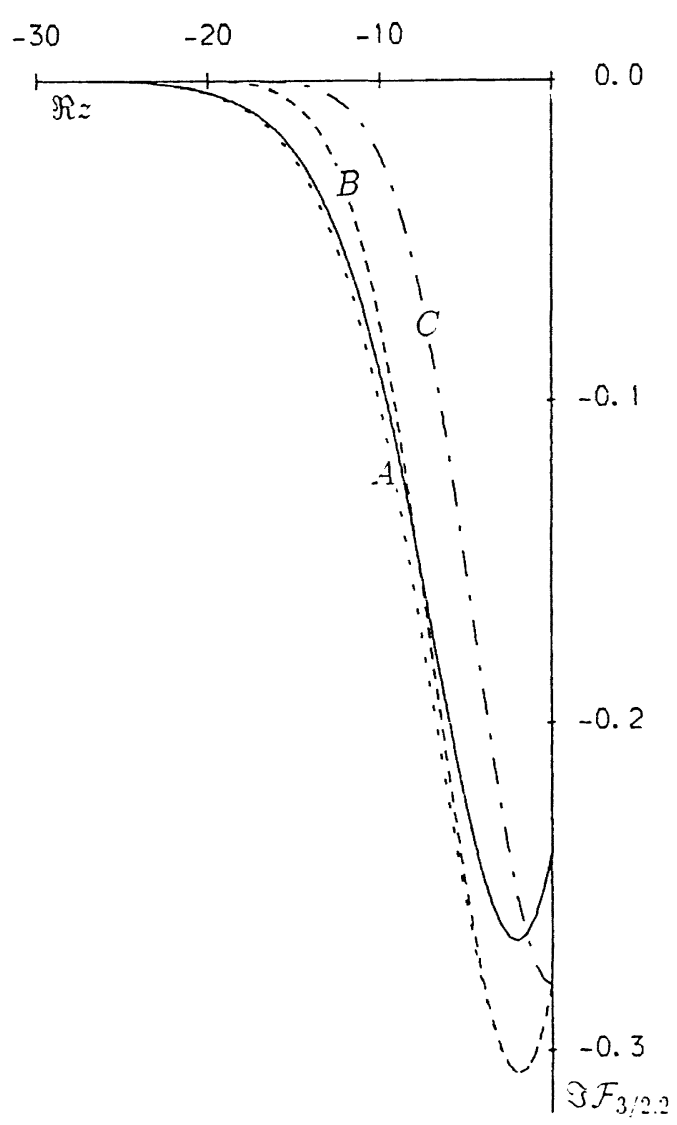

Fig. 11b. The same as Figure 3(b) but for $a=10$. 


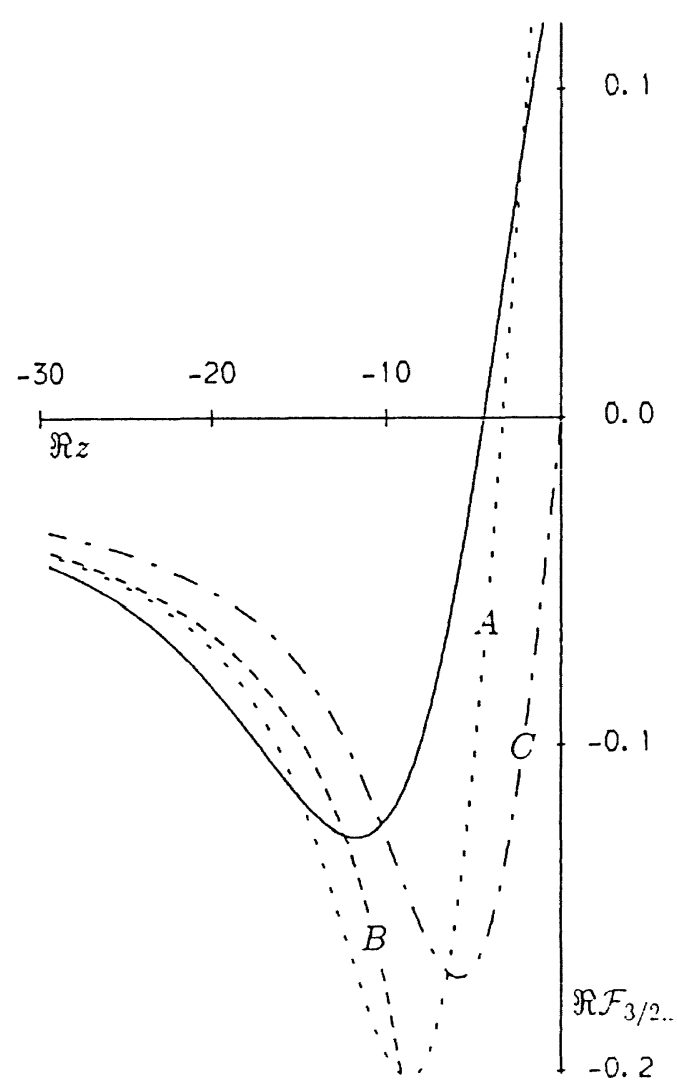

Fig. 12a. The same as Figure 4(a) but for $a=10$.

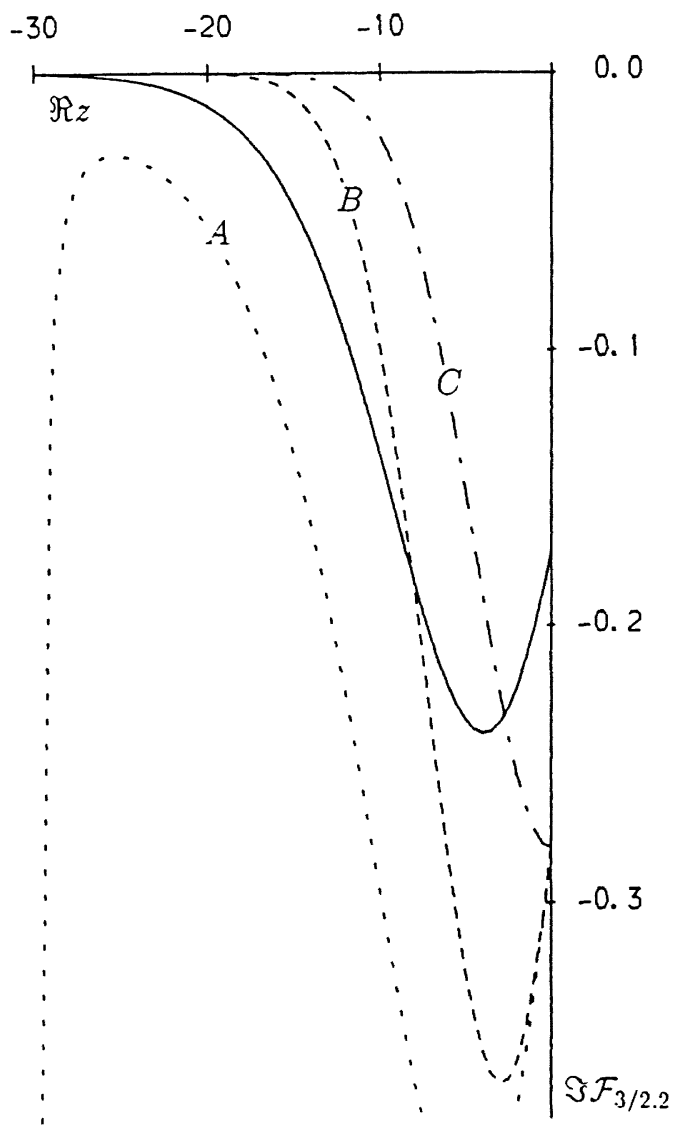

Fig. 12b. The same as Figure 4(b) but for $a=10$. 
Equation (31)) for the approximation of $\Re \mathscr{F}_{q, p}$, for $|\Re z|$ close to or greater than $a$.

(c) $\Re \mathscr{F}_{q, p}^{C}$ (see Equation (31)) can be used for qualitative analysis of $\Re \mathscr{F}_{q, p}$ except when $\Re z$ is close to zero.

(d) $\mathfrak{F}_{4, p} \mathscr{F}_{q, p}$ (see Equation (27)) is the best approximation for $\mathscr{F}_{q, p}$ when $a \gtrsim 10$ and $b=1$; however, caution is needed when using this approximation for $b=2$.

(e) $\mathfrak{F}_{q, p}^{\mathscr{F}_{q}^{B}}$ (see Equation (32)) is better than the non-relativistic expression $\mathfrak{F}_{q} \mathscr{F}_{q}^{C}$ (see Equation (33)) for almost all $\Re z$ under consideration.

\section{Acknowledgement}

One of the authors (S.S.) would like to thank SERC for financial support of this project.

\section{References}

Bahnsen, A., Jespersen, M., Neubert, T., Canu, P., Borg, H., and Frandsen, P. E.: 1985, Ann. Geophys. 3, 19.

Robinson, P. A.: 1986, J. Math. Phys. 27, 1206.

Robinson, P. A.: 1987, J. Math. Phys. 28, 1203.

Sazhin, S. S.: 1989, Phys. Scripta 40, 114.

Sazhin, S. S. and Temme, N. M.: 1990, Astrophys. Space Sci. 166, 301.

Sazhin, S. S. and Temme, N. M.: 1991, Ann. Geophys. 9, 304.

Shkarofsky, I. P.: 1966, Phys. Fluids 9, 561.

Tsai, S. T., Wu, C. S., Wang, Y. D., and Kang, S. W.: 1981, Phys. Fluids 24, 2186. 